\title{
Measuring the effects through time of the influence of visuomotor and visuotactile synchronous stimulation on a virtual body ownership illusion
}

\author{
Elena Kokkinara ${ }^{1}$, Mel Slater ${ }^{1,2,3} \S$ \\ ${ }^{1}$ EVENT Lab, Facultat de Psicologia, Universitat de Barcelona, Barcelona, Spain; \\ ${ }^{2}$ Institució Catalana Recerca i Estudis Avançats (ICREA), Universitat de Barcelona, Barcelona, \\ Spain; ${ }^{3}$ Department of Computer Science, University College London, London, UK; \\ e-mail: melslater@ub.edu \\ Received 7 June 2013, in revised form 3 January 2014
}

\begin{abstract}
Previous studies have examined the experience of owning a virtual surrogate body or body part through specific combinations of cross-modal multisensory stimulation. Both visuomotor (VM) and visuotactile (VT) synchronous stimulation have been shown to be important for inducing a body ownership illusion, each tested separately or both in combination. In this study we compared the relative importance of these two cross-modal correlations, when both are provided in the same immersive virtual reality setup and the same experiment. We systematically manipulated VT and VM contingencies in order to assess their relative role and mutual interaction. Moreover, we present a new method for measuring the induced body ownership illusion through time, by recording reports of breaks in the illusion of ownership ('breaks') throughout the experimental phase. The balance of the evidence, from both questionnaires and analysis of the breaks, suggests that while VM synchronous stimulation contributes the greatest to the attainment of the illusion, a disruption of either (through asynchronous stimulation) contributes equally to the probability of a break in the illusion.
\end{abstract}

Keywords: perceptual illusions, body ownership illusion, rubber hand illusion, multisensory integration, virtual reality

\section{Introduction}

Recent studies have demonstrated that we are able to experience the illusion that external objects are part of our body. The most well-known example of such 'body ownership illusions' is the rubber hand illusion (RHI), where it has been shown that synchronous tapping and stroking a person's hidden real arm and an aligned visible rubber arm placed in an anatomically plausible position on a table in front of the person can result in an illusion of ownership over the fake arm (Botvinick \& Cohen, 1998). The illusion is apparently caused by the synchrony of the visual and the tactile tapping, induced through the multisensory integration between what is seen (on the rubber hand) and felt on the real hand, since when the tapping is asynchronous, the illusion occurs to a much lesser extent. The RHI has also been shown to operate well in virtual reality (VR), where it has been demonstrated that participants can experience a complete virtual arm as part of their body, through passive tactile stimulation on their hidden real arm combined with synchronous visual stimulation of the visible virtual arm (Slater, Perez-Marcos, Ehrsson, \& Sanchez-Vives, 2008).

Analogously to such visuotactile (VT) correlations, synchronous visuoproprioceptive correlations during passive or active movements have also been found to induce the illusion of owning a surrogate body part (Dummer, Picot-Annand, Neal, \& Moore, 2009; Tsakiris, Prabhu, \& Haggard, 2006; Walsh, Moseley, Taylor, \& Gandevia, 2011). Moreover, the influence of agency and sensory afference on body awareness have been investigated, suggesting that proprioception (deriving from passive — that is, involuntary - movement) and 
action (deriving from active - that is, voluntary-movement), as well as touch, all constitute sources of bodily awareness (Tsakiris \& Haggard, 2005; Tsakiris et al., 2006). The illusion of ownership of a virtually presented hand has also been shown to occur on the basis of visuomotor (VM) synchrony between movements of the real hand and the virtual hand, whereas when there is asynchrony, the illusion does not occur (Sanchez-Vives, Spanlang, Frisoli, Bergamasco, \& Slater, 2010).

Combinations of sensory input from vision, touch, motor control, and proprioception are some of the mechanisms that have been shown to be the keys to body perception (for a review, see Ehrsson, 2012). Immersive VR has also been used to investigate further aspects of the illusion of ownership, while providing a full-body experience (Petkova \& Ehrsson, 2008; Slater, Spanlang, Sanchez-Vives, \& Blanke, 2010). The key to full-body ownership illusions appears to be the experience of the substitute virtual body seen through a firstperson perspective (1PP) where the participants observe the artificial/virtual body via a head-mounted display (HMD), so that they see the surrogate body substituting their own body when they look down towards themselves (Petkova, Khoshnevis, \& Ehrsson, 2011). Finally, morphological similarity to one's body has been suggested as an influence on the illusion of body ownership (Tsakiris, Carpenter, James, \& Fotopoulou, 2010; Tsakiris \& Haggard, 2005); however, the physical representation of the hand in the RHI does not necessarily need to be realistic for the illusion to take place (Longo, Schüür, Kammers, Tsakiris, \& Haggard, 2009). In a previous study, VT and visual (head-based) sensorimotor contingencies, visual perspective, and the appearance of the virtual body were systematically manipulated, in order to assess their relative importance (Maselli \& Slater, 2013).

To our knowledge, little work has been done on testing the relative importance and the possible interactions of VM and VT when both cross-modal synchronous correlations are present. Here we examine the relative contribution and mutual interaction of VM and VT stimulation on the full-body ownership illusion. We further examine whether synchronous VM feedback could cause a recalibration of the perception of incongruent VT cues and vice versa.

In order to achieve this, we carried out an experiment using VR that allowed us to integrate visual, motor, and tactile feedback. Participants were immersed in a VR scenario, where they were provided with a virtual body, seen from a 1PP. Using this setup, we were able to provide synchronous or asynchronous passive VT and active VM stimulation on the legs of the participants and thus measure and compare the resulting effect of each condition on the illusion of body ownership. More specifically, in order to assess the relative contribution of the two stimuli, we used four different groups of participants. In one group we measured the induced illusion when both touch and movement were synchronous with the visual output, in two further groups when only one of touch or movement was synchronous with vision, and in a fourth group when neither was synchronous with vision. In contrast to most other studies, we chose to deliver the stimulation on the legs in order to have the whole body within the field of view (FoV) of the participants during the simulation, thus assessing a full-body illusion, rather than just focusing on one arm.

A second purpose of this study was to test a new method to assess the illusion of ownership towards a body part. Studies of body ownership illusions have used both qualitative and quantitative measures. One standard response measurement is an ownership illusion questionnaire-for example, "I felt as if the rubber hand were my hand" or "I felt as if the virtual body was my body" (Botvinick \& Cohen, 1998; Lenggenhager, Mouthon, \& Blanke, 2009). Performance differences in localization tasks such as proprioceptive drift have been used as a quantitative response measure. In the RHI, for example, this is the distance between the felt position of the hand as blindly pointed to by the participant before and after the period of stimulation (Botvinick \& Cohen, 1998). A verbal report of the felt position of the hand judged against a ruler has also been used (Tsakiris, Haggard, Franck, Mainy, \& Sirigu, 2005; 
Tsakiris et al., 2006). Another quantitative measurement that has been used is based on the recording of physiological reactions under a threat towards the perceived body. It has been suggested that a threat to the rubber hand can cause a similar level of activity in the brain areas associated with anxiety as when the person's real hand is threatened (Ehrsson, Wiech, Weiskopf, Dolan, \& Passingham, 2007). The physiological signals that are usually recorded are skin conductance (Armel \& Ramachandran, 2003; Honma, Koyama, \& Osada, 2009; Petkova \& Ehrsson, 2008; Petkova et al., 2011; Yuan \& Steed, 2010), electrocardiogram (ECG) (Maselli \& Slater, 2013; Slater et al., 2010), changes in temperature (Hohwy \& Paton, 2010; Moseley et al., 2008), temperature sensitivity threshold (Llobera, Sanchez-Vives, \& Slater, 2013), and histamine reactivity (Barnsley et al., 2011).

Questionnaires, proprioceptive judgments, and physiological responses are normally recorded near the end of the period of stimulation, albeit often in comparison with a baseline measure recorded near the start of the experimental stimulation. Rarely is the illusion measured during the period of stimulation, exceptions being where a time course of proprioceptive judgments was measured during the stimulation (Tsakiris \& Haggard, 2005), and skin temperature was measured continuously (Moseley et al., 2008), and also the onset time that the illusion was recorded in (Perez-Marcos, Sanchez-Vives, \& Slater, 2012). Here, we introduce a new measurement technique based on data gathered throughout the stimulation period. The method is derived from a technique for measuring the illusion of presence in virtual environments, and relies on the idea of recording the moments in time when participants report loss of the illusion (Slater \& Steed, 2000). At different times during an experience the participants switch between interpreting the totality of sensory inputs as corresponding to the illusion that "the virtual body is my body", or as corresponding to the real situation that "it is just a virtual reality, with no true relationship to the real body". We call the first the 'illusion' state $(I)$ (ie that the virtual body is 'my body') and the latter the 'no illusion' state $(N)$. We counted the number of transitions from $I$ to $N$. From these data it is possible to employ a stochastic model in order to estimate the strength of the illusion. We refer to these transitions as 'breaks' in the body ownership illusion. Standard questionnaire and physiological responses (skin conductance and ECG) to a threat were also measured along with the new method.

\section{Method}

\subsection{Participants}

There were initially sixty-nine participants recruited for the experiment by advertisement around the university campus. The experiment was approved by the Comissió Bioética of the University of Barcelona. Their mean $( \pm \mathrm{SD})$ age was $22 \pm 4$ years, thirty six were female, and there was no significant difference between the 4 experimental groups mentioned above (and see section 2.4). All participants first read the instructions and basic information about the experiment and then signed an informed consent form and completed a questionnaire giving demographic information. Once the experiment was over, all participants were paid 5 euros for completing the study. Six out of sixty-nine participants were discarded due to technical failures. Another three misunderstood the procedure, failing at the training stage. All the discarded participants completed the task normally and were paid for the experiment, but their data were not used for the analysis. Hence, the final dataset consisted of sixty participants.

\subsection{Apparatus}

The participants were immersed in a VR scenario by fitting them with a stereo NVIS nVisor SX111 HMD. This has dual SXGA displays with 76 deg horizontal $\times 64$ deg vertical FoV per eye, totalling a wide FoV of 111 deg horizontal and 60 deg vertical, with a resolution of $1280 \times 1024$ per eye displayed at $60 \mathrm{~Hz}$. Head tracking was performed by a 6 degrees of 
freedom (DoF) Intersense IS-900 device. The experimenter used a 6 DoF Wand Intersense device to deliver tactile stimulation by tapping on the real legs of the participant, and controlled the stimulation with its buttons. The tracked wand was represented in the VR by a small red ball that was slaved to the movements of the real tracker, while a foam ball was attached to the wand, in order to simulate the shape of the virtual ball (figure 1). Both feet were tracked with 12 infrared Optitrack cameras, which operate at submillimetre precision (figure 2). Inverse kinematics was used to ensure that, when the participants moved their feet, the lower and upper virtual legs would move correspondingly.

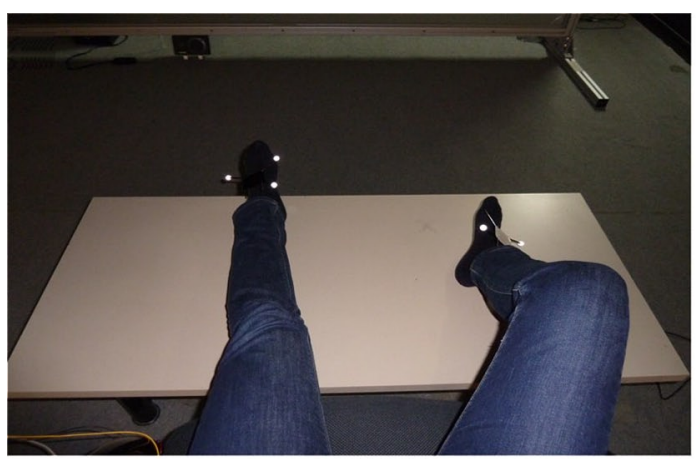

(a)

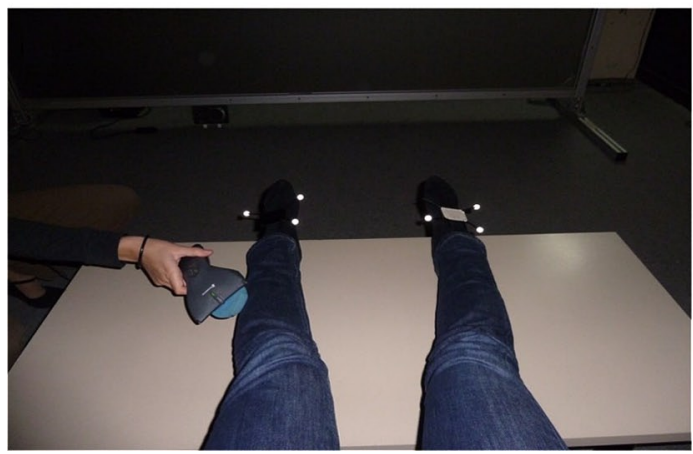

(c)

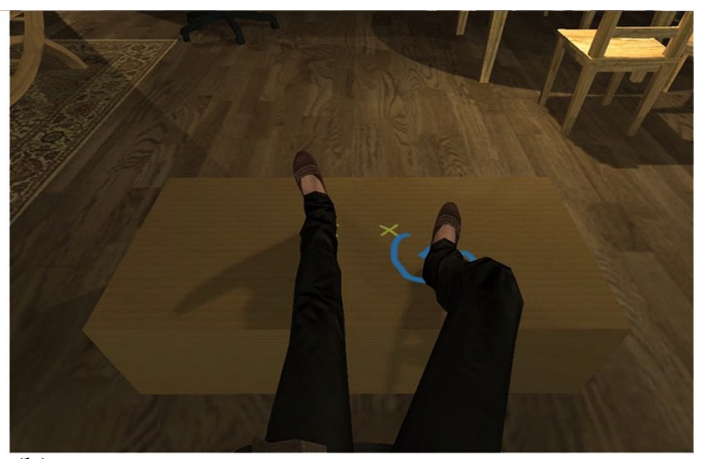

(b)

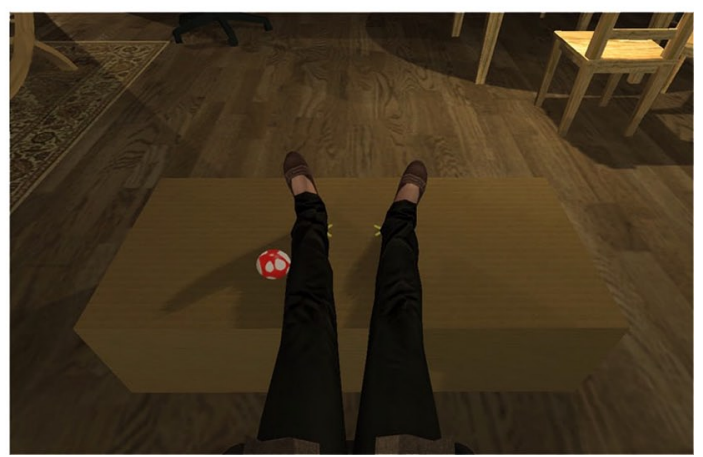

(d)

Figure 1. [In colour online, see http://dx.doi.org/10.1068/p7545] Visuomotor and visuotactile stimulation. (a) Real movement: the participant is moving according to the stimuli; (b) virtual movement: the feedback in the virtual reality might be congruent or not (prerecorded movement) with the real leg movements; (c) real tactile stimulation; the experimenter is touching the side of the leg with a tracked wand; (d) the movements of the virtual ball: synchronous with the wand's movements, or not.

The virtual environment was implemented using the Unity3D platform, and the MiddleVR ${ }^{(1)}$ plug-in was used in order to handle all 3-D tracker information and stereoscopy. The virtual model of the room was based on a Unity3D example project, and we used animationenabled models of male and female virtual bodies purchased from Rocketbox Studios. ${ }^{(2)}$

ECG and skin conductance signals were recorded at a sampling rate of $256 \mathrm{~Hz}$, using g.tec's portable biosignal acquisition device, g.MOBIlab,$+{ }^{(3)}$ while the recording and storage of the data were handled by a Simulink model in Matlab. All statistical analysis was carried out with Stata $13^{(4)}$ and RStudio(2012). ${ }^{(5)}$

(1) http://www.imin-vr.com

(2) http://www.rocketbox.de

(3) http://www.gtec.at/Products/Hardware-and-Accessories/g.MOBIlab-Specs-Features

(4) http://www.stata.com

${ }^{(5)} \mathrm{http}: / /$ www.rstudio.com/ 


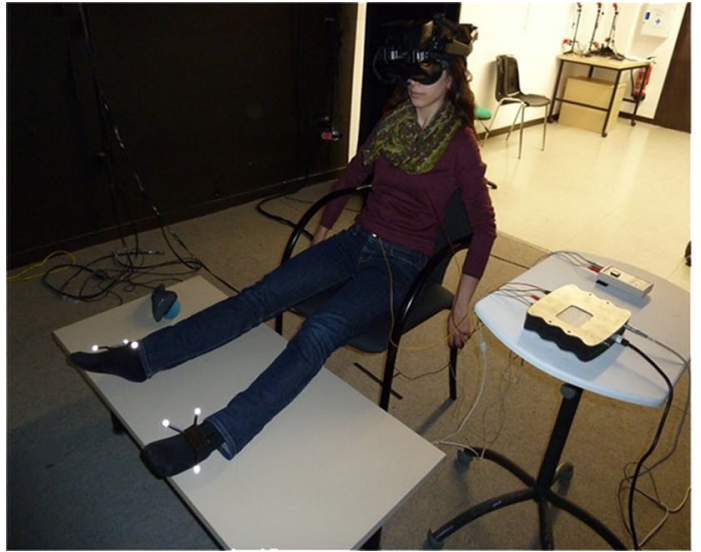

(a)

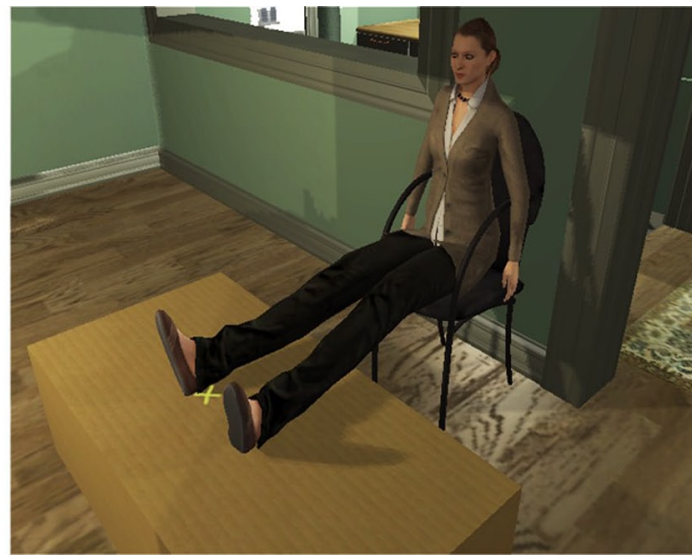

(b)

Figure 2. [In colour online.] The virtual reality setup. (a) Participants sat in the virtual reality lab, resting their legs on table. The head-mounted display provided wide field of view stereo vision and 6 degrees of freedom head tracking. Infrared trackers were attached to the feet to track the movements of the legs. Two skin conductance sensors were attached to two left hand fingers and three electrocardiogram electrodes to the main body. (b) The virtual room in which the participant was immersed. The virtual body that represented the person was positioned in a similar posture to the participant's real posture, and spatially coincident with the real body.

\subsection{Scenario}

The participants were seated on a chair in the VR laboratory, with their legs resting on a table, and with their heels placed on two marked points (figure 2). Then there was a verbal repetition of the instructions that they had previously read (section 2.1), as well as a demonstration of the motor task that they were later required to perform once immersed in the VR. After the experimenter attached the trackers for the leg movements and the sensors for recording ECG and skin conductance signals, the participant was helped to put on the HMD. It was calibrated for comfort and correct stereoscopy for each participant (Grechkin, Nguyen, Plumert, Cremer, \& Kearney, 2010). Since tracking was applied only on the head and on the two legs, we instructed the participant not to move other parts of the body. Once the virtual environment appeared, we let the participants observe the room for $30 \mathrm{~s}$ in order to familiarize themselves with the environment.

The virtual environment consisted of a room with some furniture. A gender-matched virtual body substituted the participant's real body in the same posture (figure $2 \mathrm{~b}$; see also supplementary movie at http://dx.doi.org/10.1068/p7545).

\subsection{Experimental design}

The experiment was a $2 \times 2$ factorial design with the factors VM (asynchronous, synchronous) and VT (asynchronous, synchronous). It was a between-groups design where each participant experienced only one of the four conditions with fifteen participants in each.

To provide the VM stimulation, the participants were instructed to trace a line of different shapes that would appear on the left or the right side of a virtual table (figure 1b) with their respective heel, thus executing a motor task (supplementary movie). The virtual leg would move synchronously with the real leg movements in the VM synchronous condition, whereas in the VM asynchronous condition the virtual leg would move according to a prerecorded animation. In both cases the stimulus line would disappear after $5 \mathrm{~s}$ and the participant would return the leg to the initial position. 
For the VT stimulation the experimenter tapped in a nonrhythmic pattern on the participant's real left or right leg, using the tracked wand. The participant saw a virtual ball tapping the leg. In the synchronous condition the ball would tap the leg synchronously in time and at the correct position on the leg with the tapping of the wand. In the asynchronous conditions the virtual ball tapped the leg randomly and independently of the tracking position of the wand (see supplementary movie).

The VT stimulation was administered manually by the experimenter, and VM stimulation was triggered by the experimenter pushing a button on the wand so that the stimulation line would appear on the virtual table. Hence the number of stimulations was approximately the same but not identical for each participant $(\sim 14 \mathrm{VT}$ and $\sim 14 \mathrm{VM}-$ that is, approximately 28 stimulations) over 4 minutes.

\subsection{Procedures}

Prior to starting the experiment, the participants were given the following instructions related to the elicitation of breaks in the body ownership illusion (box 1):

\section{Box 1}

\section{IMPORTANT INFORMATION: Loss of Illusion}

When you enter the virtual reality and you see the virtual body, you may have the sense that this body belongs to you. However, you may experience transitions in your sense of body ownership:

Own: sometimes you will feel that the virtual body that you are seeing is your own body.

Not own: sometimes you will become aware of your real body and that the virtual body does not belong to you. If and only whenever you experience a transition from "Own" to "Not own", please tell us "Now".

There was then a training session, which was the same for all participants, to explain the motor task (VM stimulation). Additionally, during this training we recorded the extent of the body ownership illusion under the optimal conditions - VM synchronous and VT synchronous - since the session began with sets of synchronous VM and VT stimulation, overall lasting 1 minute. To check whether an illusion of body ownership occurred, we verbally asked 5 of the 8 questions, shown in table 1 . After this we continued with further sets of VM stimulation, while deliberately introducing 5 events that we assumed would break the illusion of body ownership (see supplementary figure S1 for further information about the procedure for reporting breaks in the illusion).

After this training period, the main experiment started with alternating sets of VM and VT stimulation, which lasted overall 4 minutes. The experimenter selected one of the two types of stimulation (VT or VM) to start with, and then continued alternating between the two, until the end of the 4 minutes. In this phase the participants experienced only the combination of VM and VT stimulation according to their experimental group. For example, those in the group (VM synchronous, VT asynchronous) received only synchronous VM and asynchronous VT stimulation.

At the end of the 4 minutes of these alternating sets of VM and VT stimulation there was an event that we had designed to act as a threat to the body. This consisted of a sudden sliding of the table forward that caused the virtual legs to drop to the ground level. We expected that the physiological responses to the sudden event would be higher when the illusion of body ownership was stronger.

\subsection{Response variables}

We had three classes of response variables: (a) subjective assessment of the body ownership illusion as elicited through a questionnaire; (b) the method based on reporting of breaks in the illusion during the experience; (c) physiological responses (skin conductance and heart rate change in response to a threat towards the virtual body). 
2.6.1 Questionnaires. A postquestionnaire was designed to assess the level and quality of the illusion experienced by the participants. It was based on the questionnaire used in the original RHI paper (Botvinick \& Cohen, 1998). After the experimental trial, the participants were asked to rate 8 statements on a Likert scale from 1 (totally disagree) to 7 (totally agree). The questions are shown in table 1. Q1 referred to the feeling of where their legs were located; Q2 was concerned with the subjective strength of the ownership illusion and Q3 with the sense of motor control (agency). Q4 related to referral of touch to the virtual legs, and Q5-Q6 assessed the perceived threat towards the virtual body. Q7-Q8 were considered as control questions.

Table 1. The postexperience questionnaire. All questions were rated on a Likert scale from 1 (totally disagree) to 7 (totally agree).

\begin{tabular}{|c|c|c|}
\hline Question & Variable name & Statement \\
\hline $\mathrm{Q}^{\mathrm{a}}$ & q1location & $\begin{array}{l}\text { Overall, I felt as if my legs were located where I saw the virtual } \\
\text { legs to be. }\end{array}$ \\
\hline $\mathrm{Q}^{\mathrm{a}}$ & q2mylegs & Overall, I felt that the virtual legs were my own legs. \\
\hline $\mathrm{Q}^{\mathrm{a}}$ & q3mymovements & The movements of the virtual legs were caused by my movements. \\
\hline Q4 & q4balltouch & $\begin{array}{l}\text { It seemed as if the touch I felt was caused by the red ball touching } \\
\text { my body. }\end{array}$ \\
\hline Q5 & q5stressed & I was stressed when I saw the table being pulled away. \\
\hline Q6 & q6legsaffected & $\begin{array}{l}\text { I felt like my own legs were affected when I saw the table being } \\
\text { pulled away. }\end{array}$ \\
\hline $\mathrm{Q}^{\mathrm{a}}$ & q7morelegs & It seemed as if I might have more than two legs. \\
\hline Q8 $^{\mathrm{a}}$ & q8otherlegs & Overall, I felt that the virtual legs belonged to someone else. \\
\hline
\end{tabular}

${ }^{\text {a }}$ Indicates that the question was also asked verbally during the training period.

2.6.2 Breaks in body ownership illusion. The verbal reports of breaks were recorded with a key-press by the experimenter (though not blinded to the conditions) in response to the statement "Now" by the participant. Hence, the overall number of breaks and their time of occurrence were noted. This method resulted in two response variables: a count of the number of breaks, and a computed estimate of the strength of the illusion in the range 0 to 1 , where 0 indicates no illusion and 1 the strongest level.

The estimator of the strength in the illusion is based on a stochastic model described in Slater and Steed (2000). This model uses the simplifying assumption that the illusion is binary - that is, at any moment of time during the experience a participant can be either in the state of having the ownership illusion (state $I$ ) or not (state $N$ ). Knowing the times and the number of transitions from state $I$ to state $N$, it is possible to compute an asymptotic probability $(p)$ of being in state $I$, using a probabilistic two-state Markov chain model (Karlin, 1969; Slater \& Steed, 2000). We are able ask people to report on the transition state $I$ $\rightarrow$ state $N$ without this in itself disrupting the illusion, since when a break occurs the illusion has already been disrupted. However, it may be more problematic to ask people to report those moments corresponding to state $N \rightarrow$ state $I$ without this itself potentially disrupting the illusion (also see section 3 in supplementary material). Hence, this method relies on the participants being able to report if and when their body ownership illusion breaks.

At the end of the session the participants were given two additional questions along with the standard questionnaire. The main purpose of these was to gather information on their overall experience of the illusion. The first question asked the reason why (if it were the case) they reported no or very few transitions (ie breaks), giving four options: (1) "I rarely had the feeling that the virtual body was mine"; (2) "I almost always had the feeling that the 
virtual body was mine"; (3) "I was forgetting to report the transitions"; (4) "other reasons". No subjects reported forgetting the instruction to report transitions, and five chose "other reasons". The second question was open-ended, asking for the "causes of the transitions".

It is very important to understand that the response variable number of breaks in the illusion has a different meaning depending on the answer to the first question and that we need to consider separately the groups who answered (1) or (2), since the meaning of a 'break' is different in these two cases. Consider participants who reported a single break, for example. If they answered (2) (almost always had the illusion), this means that most of the time they had the illusion of ownership, but were disrupted once. No matter at which point in time they had this disruption, the illusion must have returned (unless the disruption was at the very end). If they answered (1) (rarely had the illusion), it meant that although there was one period when they had the illusion (probably near the start of the experience), once it was disrupted it never returned.

Similarly, in the event that no breaks were reported and the person answered (2) (almost always), then there was never a transition out of the state $I$ to the state $N$. In that case, the probability measure of the strength of the illusion would be directly assigned to 1 . On the other hand, if the person reported 0 breaks but answered (1) (rarely), then he or she was always in state $N$ and the strength of the illusion was assigned to be 0 .

2.6.3 Physiological responses. We recorded skin conductance and ECG throughout the experiment. We were particularly interested in the physiological responses caused by the threat. We expected this to be an arousing event causing stress, and therefore we would expect a skin conductance response as well as an increase in heart rate to the extent that the participants found the event disturbing. Moreover, on the basis of previous studies, these responses should be also correlated with the level of body ownership (Armel \& Ramachandran, 2003; Honma et al., 2009; Maselli \& Slater, 2013; Petkova \& Ehrsson, 2008; Petkova et al., 2011; Slater et al., 2010). Our purpose was also to find out whether these were affected by the different experimental conditions. Heart rate was calculated as the mean instantaneous heart rate (reciprocals of the response rate intervals) during a relaxation period of $10 \mathrm{~s}$ as a baseline (recorded after the training period and before the main experiment) and $10 \mathrm{~s}$ after the threat had started. Similarly, we calculated the maximum amplitude of skin conductance levels during $6 \mathrm{~s}$ of the relaxation period and $6 \mathrm{~s}$ after the threat.

\section{Results}

\subsection{Questionnaire responses}

Recall that during the training period a subset of questions from the questionnaire was asked verbally (see table 1). The results from this are presented in figure S2. This shows that, when participants experienced both VM and VT synchronous stimulation, they strongly affirmed statements associated with the illusion of body ownership, and gave very low scores on the control questions. The further advantage of this is that all participants had experienced these optimal conditions for ownership illusions, and thus were able to compare with the specific combination of VM and VT stimulation that they later experienced during the experimental phase.

Table 2a shows the medians and interquartile ranges (IQRs) of the questionnaire responses in the experimental period, from which it can be seen that the VM manipulation successfully induced agency (q3mymovements) and the VT manipulation referral of touch (q4balltouch). The sensation that the real legs were felt to be where the virtual legs were seen to be (q1location) seems to be heavily positively influenced by VM synchronous condition, and similarly for the illusion that the virtual legs were those of the participants (q2mylegs). The control questions Q7 and Q8 were low for all conditions (see also figure S3). 
To formally test these results, we used ordered logistic regression on the questionnaire scores to carry out the equivalent of two-way ANOVAs with interaction for the $2 \times 2$ experimental design. This is preferred to classic ANOVA, since the response variables are ordinal rather than measured on a continuous interval scale. For each response we first fitted the full model (main effects and interaction) and deleted the interaction term if it was not significant $(p>0.05)$, and finally deleted any main effects that were not significant. In fact none of the interaction terms was anywhere near significant. The resulting main effects significance levels are shown in table $2 \mathrm{~b}$. All of the significance levels shown except for one are very small.

Table 2. Results of the questionnaire scores.

(a) Medians (interquartile ranges-IQRs) of questionnaire responses per condition $(\mathrm{VM} \times \mathrm{VT})$

Variable Median (IQR)

\begin{tabular}{lll} 
& VT Async & VT Sync \\
\hline VM Async & $n=15$ & $n=15$ \\
q1location & $5(2)$ & $7(3)$ \\
q2mylegs & $3(2)$ & $5(3)$ \\
q3mymovements & $2(2)$ & $1(3)$ \\
q4balltouch & $1(2)$ & $7(1)$ \\
q5stressed & $2(4)$ & $2(4)$ \\
q6legsaffected & $2(4)$ & $3(4)$ \\
q7morelegs & $1(1)$ & $1(1)$ \\
q8otherlegs & $3(4)$ & $3(4)$ \\
VM Sync & $n=15$ & $n=15$ \\
q1location & $7(1)$ & $7(1)$ \\
q2mylegs & $6(1)$ & $7(1)$ \\
q3mymovements & $7(1)$ & $7(1)$ \\
q4balltouch & $3(3)$ & $7(2)$ \\
q5stressed & $2(3)$ & $4(3)$ \\
q6legsaffected & $3(3)$ & $4(5)$ \\
q7morelegs & $1(1)$ & $1(1)$ \\
q8otherlegs & $1(1)$ & $1(1)$
\end{tabular}

(b) Ordered logistic regression of questionnaire responses on VM and VT

\begin{tabular}{|c|c|c|c|c|c|c|c|c|}
\hline & VM & & & & VT & & & \\
\hline & coeffcient & SE & $Z$ & $p$ & coefficient & SE & $Z$ & $p$ \\
\hline q1location & 1.52 & 0.52 & 2.91 & 0.004 & & & & \\
\hline q2mylegs & 2.93 & 0.62 & 4.71 & 0.000 & 1.06 & 0.49 & 2.16 & 0.031 \\
\hline q3mymovements & 4.32 & 0.77 & 5.59 & 0.000 & & & & \\
\hline $\begin{array}{l}\text { q4balltouch } \\
\text { q5stressed } \\
\text { q6legsaffected } \\
\text { q7morelegs }\end{array}$ & & & & & 3.28 & 0.64 & 5.16 & 0.000 \\
\hline q8otherlegs & -2.26 & 0.55 & -4.09 & 0.000 & & & & \\
\hline
\end{tabular}

Note: $p=0.000$ means $p<0.0005$; nonsignificant terms are blank; $\mathrm{SE}=$ standard error. 


\subsection{Overall body ownership}

Table 3 presents the breakdown of responses to the question about the reason for the number of breaks in the illusion, which addresses the overall illusion of body ownership. The majority of those who reported that they almost always had the illusion of owning the virtual body (I) immediately after the experiment were in the VM synchronous group (twenty eight out of thirty seven). Amongst those who reported that they rarely had the feeling of the body ownership illusion $(N)$, seventeen out of eighteen were in the VM asynchronous group and thirteen out of eighteen were in the VT asynchronous group. There were five who did not answer either the $I$ or the $N$ category, but rather "other".

We can transform this response variable $(y)$ into a binary one. Ignoring the 5 'other' responses, we take the score $y$ as 1 when the answer is $I$ and 0 when the answer is $N$. Hence, in the normal terminology of binomial logistic regression ' 1 ' (almost always body ownership) is a 'success' and '0' (rarely body ownership) is a 'failure'. We regress $y$ on the two factors VM and VT. (Note that the results are almost identical whether robust estimates of standard errors are used or default standard errors-using the options in Stata 13.) The results show no interaction effect, but significant main effects for VM (coefficient estimate $\pm \mathrm{SE}$ of coefficient estimate $=4.54 \pm 1.23, z=3.68, p<0.0005)$ and VT $(2.23 \pm 0.94, z=2.38, p=0.017)$. The Pearson goodness of fit test has $\chi_{1}^{2}=0.14, p>0.71$, indicating a good fit. In fact, the fit leads to an $85 \%$ correct classification of the original data.

From the logistic model we can compute the estimated probabilities of 'success' for each individual. The histogram of these estimated probabilities is shown in figure 3 , where it can be seen that the probabilities fall into three clusters. It turns out that all participants in the cluster around $0.2(n=14)$ had experienced both VM and VT stimulation asynchronously. All participants in the cluster between 0.6 and $0.8(n=12)$ had experienced VM stimulation asynchronously and VT synchronously. Finally, in the cluster with the highest probability estimates $(n=29)$, all had experienced VM stimulation synchronously, whereas fifteen out of twenty nine had experienced VT stimulation synchronously. In other words, for those in the highest probability group in our sample, it is certain that they had experienced VM stimulation synchronously, but only a $52 \%$ chance of having experienced VT stimulation synchronously. Another way to put this is that all those with synchronous VM stimulation were in the highest probability cluster, and all those with asynchronous VM stimulation were not in the highest probability cluster (excluding participants in the 'other' group). Thus, VM alone is sufficient to predict whether or not an individual falls into the highest probability cluster. It would appear, therefore, that VM plays the determining role in the generation of this body ownership illusion.

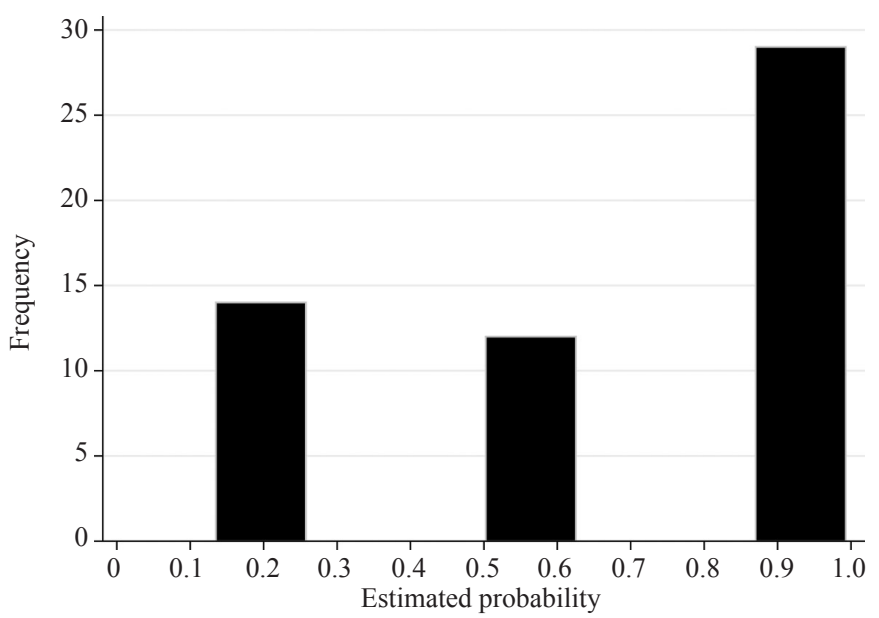

Figure 3. Histogram of the estimated probabilities of 'success' from the binary logistic regression. 


\subsection{Breaks in body ownership illusion}

The above analysis considers what contributed to the ownership illusion. Analysis of the numbers of breaks will help to understand the balance of factors that tended to disrupt it. Table 3 shows the means of the numbers of breaks. Considering the $I$ group, it is clear that there is no important change when moving from both VM and VT asynchronous to either one being synchronous. However, when both are synchronous there is a strong decrease of about 10 breaks in both cases, leading to 0 breaks when both are synchronous.

Table 3. Mean and standard errors of numbers of breaks, mean intervals between breaks (s), estimated probability of the illusion, and frequency of responses to the question about reasons for breaks, by condition.

\begin{tabular}{|c|c|c|}
\hline Reason & VT Async & VT Sync \\
\hline \multicolumn{3}{|c|}{ (N) Almost never had the illusion } \\
\hline \multicolumn{3}{|c|}{ VM Async } \\
\hline Number of breaks & $6.7 \pm 2.04$ & $11.2 \pm 2.89$ \\
\hline Interval & $10.8 \pm 2.19$ & $61.7 \pm 44.59$ \\
\hline Probability of illusion $(p)$ & $0.11 \pm 0.03$ & $0.18 \pm 0.05$ \\
\hline$n$ & 12 & 5 \\
\hline \multicolumn{3}{|l|}{ VM Sync } \\
\hline Number of breaks & 0 & - \\
\hline Interval & - & - \\
\hline Probability of illusion $(p)$ & 0 & - \\
\hline$n$ & 1 & 0 \\
\hline \multicolumn{3}{|c|}{ (I) Almost always had the illusion } \\
\hline \multicolumn{3}{|c|}{ VM Async } \\
\hline Number of breaks & $16.5 \pm 3.50$ & $10.3 \pm 1.80$ \\
\hline Interval & $15.0 \pm 2.88$ & $22.9 \pm 3.58$ \\
\hline Probability of illusion $(p)$ & $0.73 \pm 0.06$ & $0.83 \pm 0.03$ \\
\hline$n$ & 2 & 7 \\
\hline \multicolumn{3}{|l|}{ VM Sync } \\
\hline Number of breaks & $9.7 \pm 2.31$ & $0 \pm 0$ \\
\hline Interval & $86.7 \pm 29.6$ & - \\
\hline Probability of illusion $(p)$ & $0.84 \pm 0.04$ & $1 \pm 0$ \\
\hline$n$ & 13 & 15 \\
\hline \multicolumn{3}{|l|}{ Other } \\
\hline \multicolumn{3}{|l|}{ VM Async } \\
\hline Number of breaks & 12 & $12.3 \pm 0.88$ \\
\hline Interval & 17.7 & $19.5 \pm 1.52$ \\
\hline Probability of illusion $(p)$ & - & - \\
\hline$n$ & 1 & 3 \\
\hline \multicolumn{3}{|l|}{ VM Sync } \\
\hline Number of breaks & 13 & - \\
\hline Interval & 18.1 & - \\
\hline Probability of illusion $(p)$ & - & - \\
\hline$n$ & 1 & 0 \\
\hline
\end{tabular}

Regression of the number of breaks on VM and VT for the $I$ group $(n=37)$ shows that there is no interaction, but the main effects are highly significant (table 4). (The residual errors of the fit are compatible with normality, Shapiro-Wilk $p=0.34$.) The effect sizes, partial $\eta^{2}$, are also substantial. The coefficients of approximately -9 fit well with what is observed in table 3 . Therefore, for those who almost always had the illusion, a break in the illusion was associated with VM and VT to about the same degree. 
Table 4. Regression of number of breaks on (VM, VT) ( 0 asynchronous, 1 synchronous) for the $I$ group (almost always had the illusion).

\begin{tabular}{llllll}
\hline Term & Coefficient & SE & $t$ & $p$ & Partial $\eta^{2}$ \\
\hline Constant & 18.7 & 2.32 & 8.06 & $<0.0005$ & \\
Visuomotor (VM) & -9.4 & 2.13 & -4.39 & $<0.0005$ & 0.36 \\
Visuotactile (VT) & -9.1 & 1.86 & -4.86 & $<0.0005$ & 0.41 \\
Note: $R^{2}=0.51, F_{2,34}=17.72, p<0.00005(n=37) ; \mathrm{SE}=$ standard error. & \\
\hline
\end{tabular}

We cannot carry out a similar regression analysis for those in the $N$ group (rarely had the illusion), since there is only 1 entry in the VM synchronous condition. However, it can be seen that, when VM is asynchronous, VT synchronous increases the number of breaks, and also increases the mean interval between them. This is in line with the different interpretation of a break in this condition (here, more breaks associated with greater interval between them indicates overall a greater degree of illusion).

In Slater and Steed (2000) it was shown how to compute estimated probabilities of experiencing the illusion based on the numbers of breaks (see section 3 in supplementary material). The means and standard errors of these probabilities $(p)$ are shown in table 3 . The 5 cases where the participants gave the response 'other' in the question about the reason of few or no break were ignored. Regression of the probabilities $(p)$ on VM and VT shows no interaction effect but significant main effects, shown in table 5 (Shapiro-Wilk $p=0.09$ ).

Table 5. Regression of probability of illusion ( $p$ ) on (VM,VT) ( 0 asynchronous, 1 synchronous).

\begin{tabular}{llllll}
\hline Term & Coefficient & SE & $t$ & $p$ & Partial $\eta^{2}$ \\
\hline Constant & 0.23 & 0.06 & 4.09 & $<0.0005$ & \\
Visuomotor (VM) & 0.52 & 0.07 & 7.82 & $<0.0005$ & 0.54 \\
Visuotactile (VT) & 0.29 & 0.07 & 4.35 & $<0.0005$ & 0.27 \\
Note: $R^{2}=0.62, F_{2,52}=42.07, p<0.00005(n=55) ; \mathrm{SE}=$ standard error. & \\
\hline
\end{tabular}

In spite of the quite different way that these quantities $(p)$ were derived, the estimated probability of the illusion is also strongly positively correlated with Q1 (self-localization), Q2 (ownership), Q3 (agency), Q4 (referral of touch) ( $r=0.44, p=0.0007 ; r=0.76, p<0.00005$; $r=0.73, p<0.00005 ; r=0.39, p=0.004$, respectively), whereas a negative correlation was found with the control question on ownership Q8 $(r=-0.69, p<0.00005)$. The correlations with questions Q5, Q6 (assessment of stress levels), and Q7 (control question) were not significant ( $r=-0.01, p=0.95 ; r=0.05, p=0.70 ; r=-0.13, p=0.33$, respectively).

Table S1 (supplementary material) illustrates some of the characteristic answers to the open question about the 'causes of the breaks'.

\subsection{Analysis of the physiological responses}

The skin conductance levels were averaged across all subjects (figure 4). A response can be seen in the few seconds after the threat (time $>0$ ). To compare the responses across the conditions, we used as a response variable the percentage of change between the maximum skin conductance amplitude in the $6 \mathrm{~s}$ baseline period and in the $2-8 \mathrm{~s}$ period after the threat (SCchange). We found a positive correlation between each of Q5 and Q6 (subjective assessment of stress) and SC Change ( $r=0.32, p=0.014$ and $r=0.27, p=0.044$, respectively) (see figure 5). This serves as a validation between the physiological response and the questionnaire variables, indicating that the event of the table moving away was arousing. 


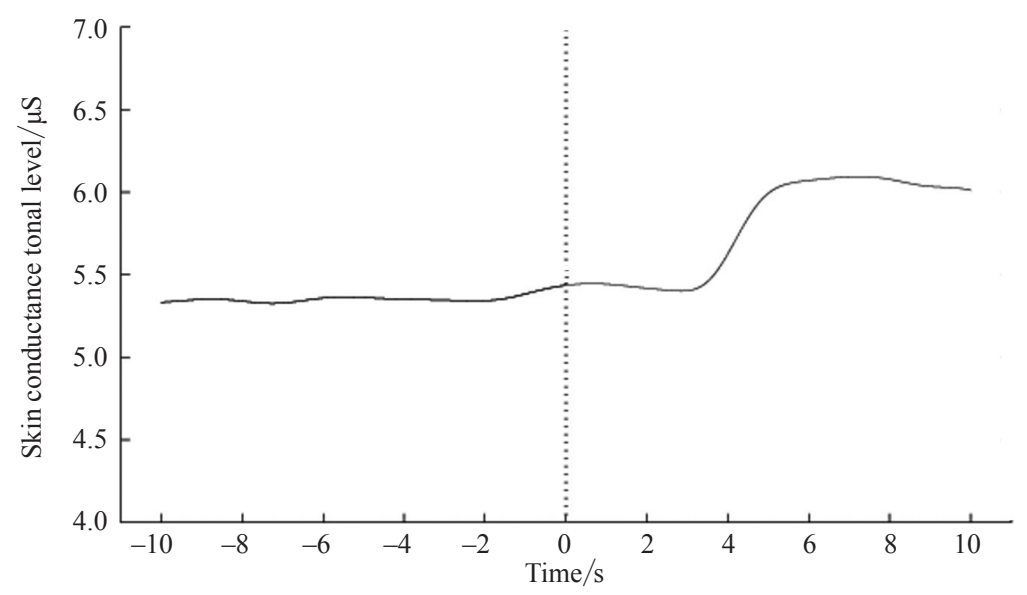

Figure 4. Skin conductance levels averaged over all participants $10 \mathrm{~s}$ before and after the threat (dashed line at time $0 \mathrm{~s}$ ).
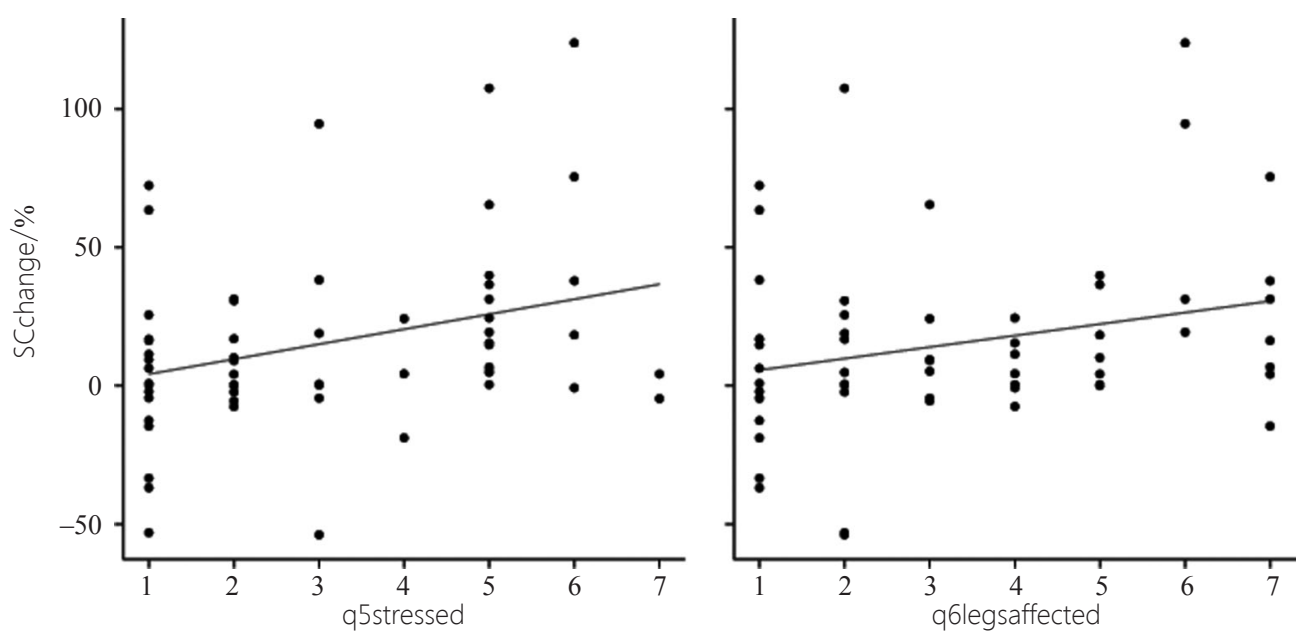

Figure 5. Scatterplot of the percentage of change between the maximum skin conductance amplitude in the $6 \mathrm{~s}$ baseline period and in the $2-8 \mathrm{~s}$ period after the threat (SCchange) and q5stressed (left) and q6legsaffected (right).

However, this event seems to have been arousing under all experimental conditions, since there are no specific effects of the VM or VT conditions on this measure.

The mean $( \pm \mathrm{SD})$ instantaneous heart rate in the $10 \mathrm{~s}$ baseline (relaxation) period (BaselineHR) was $72 \pm 13.5 \mathrm{bpm}$, and in the $10 \mathrm{~s}$ period after the threat had started (HR) it was $76 \pm 11.8 \mathrm{bpm}(n=60)$. A paired $t$-test shows that the difference is significant $\left(t_{59}=5, p<0.00005\right)$ (two-sided). In combination with the change in skin conductance, this indicates that the threat event was effective. Moreover, the skin conductance amplitude and the change in heart rate from baseline to threat are positively correlated $(r=0.29$, $p=0.025)$.

\section{Discussion}

Earlier results from comparison of the effects of VM with VT correlations on body ownership illusions have been quite diverse. Previous studies have shown that there are reports of similar levels of ownership from passive VT conditions (ie stroking by the experimenter) and active movement (ie where the participant voluntarily moves part of the body), each tested separately (Dummer et al., 2009; Tsakiris et al., 2006; Walsh et al., 2011). However, active VT stimulation 
incorporates both touch and movement in coordination, since one needs to move in order to voluntarily touch an object. There are indications that active compared with passive touching conditions both induce similar body ownership responses towards a virtual arm (Pabon et al., 2010). Similar responses for active congruent VT correlations have been found when compared with incongruent ones; although the movements of the virtual hand were congruent with those of the real hand, the virtual hand was not seen to touch a virtual object, even while the real hand was touching a real one (Kilteni, Normand, Sanchez-Vives, \& Slater, 2012) - and, even so, the illusion of ownership over the virtual hand was maintained. However, when active synchronous VT stimulation along with 1PP was shown to induce a strong ownership illusion of a larger belly, the equivalent asynchronous condition (using incongruent movements and incongruent VT feedback) failed in this (Normand, Giannopoulos, Spanlang, \& Slater, 2011). These two studies included both VT and VM stimulation under the same scenario using active tactile stimulation. However, the two stimuli were not inseparable or independently manipulated, since touch was a result of movement so that there was no way to distinguish their separate influence.

In our study we were able to manipulate the two stimuli independently. The results provide evidence that congruent multisensory and sensorimotor feedback between the unseen real and the seen virtual legs can induce sensations that the seen legs are part of the actual body. Moreover, our findings suggest that the production of the illusion is more strongly and positively influenced by congruent VM correlations than VT. However, the illusion can be broken to the same extent by incongruent VM or incongruent VT stimulation. This distinction between what contributes to the illusion of body ownership compared with what breaks the illusion does not appear to have been studied before.

The results from questionnaires and the analysis of breaks suggest that asynchronous VT may be discounted when synchronous VM cues are provided. For example, we can predict a high or low estimated probability of the illusion solely from knowing which VM group (synchronous or asynchronous) the person was in. Although we used a different setup to apply and manipulate VM and VT congruencies, this result supports the finding of Kilteni et al. (2012), where incongruent VT feedback was neglected when synchronous VM stimulation was provided. High levels of ownership can be also induced under incongruent VM feedback, when VT correlations are present, yet the evidence does not support the notion that VM asynchronous stimulation can be discounted (see q2mylegs in figure S3). Finally, asynchronous VT stimulation combined with asynchronous VM stimulation is shown to be incompatible with the illusion.

In contrast to previous studies, here all participants experienced full-body ownership through congruent multimodal stimulation during the training session. We believe that this can provide a grounding against which participants evaluate the illusion associated with the various incongruent conditions. Moreover, through doing this, it is possible to avoid the bias likely introduced when participants experience first an incongruent condition (for example, in a counterbalanced within-groups experimental design) and are asked to rate the illusion without any prior experience of what it is that they are rating. That this can give rise to highly significant bias is shown in Llobera et al. (2013).

The results of the impact of VM and VT stimulation on the illusion of ownership are supported by the balance of the evidence from the questionnaires as well as from the analysis of breaks. Moreover, as expected, only VM stimulation seems to affect agency and selflocalization, whereas only VT affects the referral of touch. Overall, we found no interaction effects between the two factors. See also the boxplots in figure S3 in the supplementary material for a comparison of the score distributions across the four conditions in each question. 
With hindsight, it is clear that the nature of the threat (the table suddenly moving away) was not one that would differentiate ownership levels between the four conditions of the experiment, since it was perceived as an arousing event (as indicated by the skin conductance and heart rate change) independently of the experimental factors. Its utility is that it did provide further evidence for the validity for the experiment, since the skin conductance response was correlated with the subjective indication of stress as measured by Q5. However, this result is not necessarily related to ownership, since skin conductance levels could rise in response to any arousing event. It is more likely to be related to presence (Sanchez-Vives $\&$ Slater, 2005). Another argument for the similar physiological responses across conditions could be that, since the threatening event occurred 1-3 s after the last stimulation, the illusion of ownership could have emerged in the absence of other stimulation solely due to the 1PP with respect to the static colocated body.

Previous studies have mainly based their results on self-reports, perceptual judgments, and behaviours, as measured after the stimulation period. These measurements could be biased by the very last impression of the experimental phase, rather than based on the overall experience. The results of this study were evaluated also using a new methodology for measuring the illusion of body ownership in VR throughout the stimulation period. We customized the earlier method that was used as a presence measure (Slater \& Steed, 2000). The correlation between breaks and questionnaire responses, elicited in quite different ways, also points towards a consistency between the different types of measures.

Acknowledgments. This research was funded by the FP7 EU VR-HYPERSPACE (AAT-285681) project funded under the Aeronautics and Air Transport work programme. We would like to thank Jorge Arroyo Palacios for helping with the skin conductance and ECG recordings and analysis and Gemma Boira Fuentes for helping with the experiments.

\section{References}

Armel, K. C., \& Ramachandran, V. S. (2003). Projecting sensations to external objects: evidence from skin conductance response. Proceedings. Biological Sciences/The Royal Society, 270(1523), 1499-1506.

Barnsley, N., McAuley, J. H., Mohan, R., Dey, A., Thomas, P., \& Moseley, G. L. (2011). The rubber hand illusion increases histamine reactivity in the real arm. Current Biology, 21(23), R945-R946.

Botvinick, M., \& Cohen, J. (1998). Rubber hands 'feel' touch that eyes see. Nature, 391(6669), 756.

Dummer, T., Picot-Annand, A., Neal, T., \& Moore, C. (2009). Movement and the rubber hand illusion. Perception, 38, 271-280.

Ehrsson, H. H. (2012). The concept of body ownership and its relation to multisensory integration. In B. E. Stein (Ed.), The new handbook of multisensory processing (pp. 775-792). Cambridge, MA: MIT Press.

Ehrsson, H. H., Wiech, K., Weiskopf, N., Dolan, R. J., \& Passingham, R. E. (2007). Threatening a rubber hand that you feel is yours elicits a cortical anxiety response. Proceedings of the National Academy of Sciences of the USA, 104, 9828-9833.

Grechkin, T. Y., Nguyen, T. D., Plumert, J. M., Cremer, J. F., \& Kearney, J. K. (2010). How does presentation method and measurement protocol affect distance estimation in real and virtual environments? ACM Transactions on Applied Perception, 7(4), 26:1-26:18.

Hohwy, J., \& Paton, B. (2010). Explaining away the body: Experiences of supernaturally caused touch and touch on non-hand objects within the rubber hand illusion. PLOS ONE, 5(2), e941.

Honma, M., Koyama, S., \& Osada, Y. (2009). Double tactile sensations evoked by a single visual stimulus on a rubber hand. Neuroscience Research, 65, 307-311.

Karlin, S. (1969). A first course in stochastic processes. San Diego, CA: Academic Press.

Kilteni, K., Normand, J.-M., Sanchez-Vives, M. V., \& Slater, M. (2012). Extending body space in immersive virtual reality: A very long arm illusion. PLoS ONE, 7(7), e40867.

Lenggenhager, B., Mouthon, M., \& Blanke, O. (2009). Spatial aspects of bodily self-consciousness. Consciousness and Cognition, 18, 110-117. 
Llobera, J., Sanchez-Vives, M. V., \& Slater, M. (2013). The relationship between virtual body ownership and temperature sensitivity. Journal of the Royal Society Interface, 10(85), 20130300.

Longo, M. R., Schüür, F., Kammers, M. P. M., Tsakiris, M., \& Haggard, P. (2009). Self awareness and the body image. Acta Psychologica, 132, 166-172.

Maselli, A., \& Slater, M. (2013). The building blocks of the full body ownership illusion. Frontiers in Human Neuroscience, 7(83), 1-15.

Moseley, G. L., Olthof, N., Venema, A., Don, S., Wijers, M., Gallace, A., \& Spence, C. (2008). Psychologically induced cooling of a specific body part caused by the illusory ownership of an artificial counterpart. Proceedings of the National Academy of Sciences of the USA, 105, 13169-13173.

Normand, J.-M., Giannopoulos, E., Spanlang, B., \& Slater, M. (2011). Multisensory stimulation can induce an illusion of larger belly size in immersive virtual reality. PLOS ONE, 6(1), e16128.

Pabon, S., Padilla, M. A., Frisoli, A., Greco, G., Bergamasco, M., \& Dataglove, A. P. (2010). Ownership illusion of the hand/arm through dynamic VR interactions and automatic vibrotactile stimulation. 19th IEEE International Conference on Robot and Human Interactive Communication, Viareggio, Italy, 13-15 September (pp. 629-634). Piscataway, NJ: IEEE Publications.

Perez-Marcos, D., Sanchez-Vives, M. V., \& Slater, M. (2012). Is my hand connected to my body? The impact of body continuity and arm alignment on the virtual hand illusion. Cognitive Neurodynamics, 6, 295-305.

Petkova, V. I., \& Ehrsson, H. H. (2008). If I were you: Perceptual illusion of body swapping. PLoS ONE, 3(12), e3832.

Petkova, V. I., Khoshnevis, M., \& Ehrsson, H. H. (2011). The perspective matters! Multisensory integration in ego-centric reference frames determines full-body ownership. Frontiers in Psychology, 2(35), 1-7.

Sanchez-Vives, M. V., \& Slater, M. (2005). From presence to consciousness through virtual reality. Nature Reviews Neuroscience, 6, 332-339.

Sanchez-Vives, M. V., Spanlang, B., Frisoli, A., Bergamasco, M., \& Slater, M. (2010). Virtual hand illusion induced by visuomotor correlations. PLOS ONE, 5(4), e10381.

Slater, M., Perez-Marcos, D., Ehrsson, H. H., \& Sanchez-Vives, M. V. (2008). Towards a digital body: The virtual arm illusion. Frontiers in Human Neuroscience, 2(6), 1-8.

Slater, M., Spanlang, B., Sanchez-Vives, M. V., \& Blanke, O. (2010). First person experience of body transfer in virtual reality. PLoS ONE, 5(5), e10564.

Slater, M., \& Steed, A. (2000). A virtual presence counter. Presence: Teleoperators and Virtual Environments, 9, 413-434.

Tsakiris, M., Carpenter, L., James, D., \& Fotopoulou, A. (2010). Hands only illusion: Multisensory integration elicits sense of ownership for body parts but not for non-corporeal objects. Experimental Brain Research, 204, 343-352.

Tsakiris, M., \& Haggard, P. (2005). The rubber hand illusion revisited: visuotactile integration and self-attribution. Journal of Experimental Psychology: Human Perception and Performance, 31, $80-91$.

Tsakiris, M., Haggard, P., Franck, N., Mainy, N., \& Sirigu, A. (2005). A specific role for efferent information in self-recognition. Cognition, 96, 215-231.

Tsakiris, M., Prabhu, G., \& Haggard, P. (2006). Having a body versus moving your body: How agency structures body-ownership. Consciousness and Cognition, 15, 423-432

Walsh, L. D., Moseley, G. L., Taylor, J. L., \& Gandevia, S. C. (2011). Proprioceptive signals contribute to the sense of body ownership. The Journal of Physiology, 589, 3009-3021.

Yuan, Y., \& Steed, A. (2010). Is the rubber hand illusion induced by immersive virtual reality? IEEE Virtual Reality 2010 Conference, Waltham, MA, 20-24 March (pp. 95-102). Piscataway, NJ: IEEE Publications. 